\title{
A FEW REMARKS ON VARIATIONAL MODELS FOR DENOISING*
}

\author{
RUSTUM CHOKSI ${ }^{\dagger}$, IRENE FONSECA ${ }^{\ddagger}$, AND BARBARA ZWICKNAGL ${ }^{\S}$
}

\begin{abstract}
Variational models for image and signal denoising are based on the minimization of energy functionals consisting of a fidelity term together with higher-order regularization. In addition to the choices of function spaces to measure fidelity and impose regularization, different scaling exponents appear. In this note we present a few simple remarks on (i) the stability with respect to deterministic noise perturbations, captured via oscillatory sequences converging weakly to zero, and (ii) exact reconstruction.
\end{abstract}

Key words. Denoising, higher-order regularization, stability, weak convergence, Brezis-Lieb condition.

AMS subject classifications. $\quad 49 \mathrm{~N} 45,94 \mathrm{~A} 08$.

\section{Introduction}

In recent years, a wealth of research has been dedicated to the study of higherorder regularized variational methods for image and signal denoising. The idea of using a higher-order regularization to overcome ill-posedness in variational methods associated with ill-posed inverse problems is well-known, and it dates back to Andrey Tikhonov ([33]). Since the pioneering work of Rudin, Osher, and Fatemi [31], much attention has been devoted to $T V$-regularization in the context of image denoising. Analytical work has mainly targeted the study of fast and efficient numerical implementations and comparison with other models (see [11, 15, 17, 24, 25, 28] and the references therein), the understanding of the structure of possible minimizers (see $[1,2,3,4,6,7,8,13,14,16,18,19,22,27,30,34])$, and the analysis of the effects of $T V$-regularization in terms of edge, block, texture, and scale preservation (see $[20,25,27,32])$.

Here we will consider the following class of higher-order regularized variational models for denoising. Given a noisy measured signal $f: \Omega \rightarrow \mathbb{R}$ in a domain $\Omega \subset \mathbb{R}^{N}$, with

$$
f=f_{0}+h
$$

where $f_{0}$ is the undisturbed signal and $h$ is a noise sample, we seek to reconstruct the original signal $f_{0}$, or at least a good approximation of it, from the measured signal $f$. A standard technique for such problems is to minimize functionals of the form

$$
J(u):=|u|_{\mathcal{X}}^{\alpha}+\lambda\|u-f\|_{\mathcal{Y}}^{\beta}, \quad u: \Omega \rightarrow \mathbb{R},
$$

with $\mathcal{X}$ and $\mathcal{Y}$ Banach function spaces such that, typically, $\mathcal{X} \subset \mathcal{Y}$, and $\alpha, \beta \geq 1$. The space $\mathcal{Y}$ is often a Lebesgue space $L^{p}(\Omega)$ for some $1 \leq p<\infty$, but it may also be a normed space with a weaker norm such as the $H^{-1}$ or the $G$-norm of Meyer (see [27]). These weaker norms have proven to be successful when one wishes to retain textures

${ }^{*}$ Received: November 27, 2012; accepted (in revised form): July 7, 2013. Communicated by Luminita Vese.

${ }^{\dagger}$ Department of Mathematics and Statistics, McGill University, Montreal, Canada (rchoksi@ math.mcgill.ca).

${ }^{\ddagger}$ Department of Mathematical Sciences, Carnegie Mellon University, Pittsburgh, USA (fonseca@ andrew.cmu.edu).

§Institut für Angewandte Mathematik, Universität Bonn, Germany (zwicknagl@iam.uni-bonn.de). 
in images (see $[25,27,28]$ ). The most popular choices for the function space $\mathcal{X}$ include the space $B V(\Omega)$ of functions of bounded variation, and Sobolev spaces $W^{2, k}(\Omega)$ for some $k \geq 1$. Having these examples in mind, we assume that $\mathcal{X}$ is equipped with a norm $\|\cdot\|_{\mathcal{X}}$ and a semi-norm $|\cdot|_{\mathcal{X}}$, where the latter is sequentially lower semicontinuous with respect to an appropriate weak convergence in $\mathcal{X}$, and such that for some $C>0$

$$
\|u\|_{\mathcal{X}} \leq C\left(|u|_{\mathcal{X}}+\|u\|_{\mathcal{Y}}\right) \quad \text { for all } u \in \mathcal{X}
$$

The second term in (1.2) is called the fidelity term and it encourages minimizers to stay close to the observed signal. The first term is intended to be a regularization which should filter out (oscillatory) noisy parts of the observed image. The two terms are weighted by a regularization parameter $\lambda>0$. The exponents $\alpha$ and $\beta$ are usually natural numbers. Typical examples of such functionals include the total variation based regularization in the Rudin-Osher-Fatemi (ROF) model [31] on two-dimensional bounded Lipschitz domains $\Omega \subset \mathbb{R}^{2}$, namely

$$
J(u):=|u|_{B V(\Omega)}+\lambda\|u-f\|_{L^{2}(\Omega)}^{2},
$$

for $u \in L_{l o c}^{1}(\Omega)$, with the $B V$-semi-norm ${ }^{1}$ defined by (2.2), or variants with slightly different fidelity terms, such as

$$
J(u):=|u|_{B V(\Omega)}+\lambda\|u-f\|_{L^{2}(\Omega)}, \quad \text { or } \quad J(u):=|u|_{B V(\Omega)}+\lambda\|u-f\|_{L^{1}(\Omega)} .
$$

These functionals share a common feature: If we input for $f$ a highly oscillatory perturbation of a clean image $f_{0}$, minimization returns a signal which is close to $f$ (the influence of the second term) in a way as to minimize oscillations (the influence of the first term). Not surprisingly, numerical simulations of minimizers for a noisy perturbation of clean images result in states closer to the actual image than the measured signal. In this way, we say that the original measured signal $f$ has been denoised. We refer to the book of Chan and Shen [17], and the references therein, for various examples and types of noise perturbations.

It is therefore natural to assess two properties of these variational problems. The first is their stability with respect to weakly vanishing noise. Precisely, if

$$
J_{n}(u):=|u|_{\mathcal{X}}^{\alpha}+\lambda\left\|u-f-h_{n}\right\|_{\mathcal{Y}}^{\beta} \quad \text { and } J(u):=|u|_{\mathcal{X}}^{\alpha}+\lambda\|u-f\|_{\mathcal{Y}}^{\beta},
$$

where the sequence of noise $\left\{h_{n}\right\}$ converges to zero in some weak sense, then the minimizers of the disturbed functionals $J_{n}$ should converge to minimizers of the undisturbed functional $J$. In fact, one would expect that $\left\{J_{n}\right\} \Gamma$-converges to $J$ (see Definition 2.2). The fact that functionals of type (1.2) are stable with respect to small perturbations in the strong norm is easy to establish, and has been noted in [1]. However, the paradigm of these variational problems is that minimization of the regularization reduces highly oscillatory perturbations of the clean signal which one often attributes to noise. Thus it is natural to consider perturbations which do not go to zero in a strong sense but rather in a weak sense, for example increasing oscillations between two fixed values. Surprisingly, this notion of stability with respect to weak perturbations has rarely been addressed. In fact, we are aware of only [19] and [21] $]^{2}$.

\footnotetext{
${ }^{1}$ Sometimes the regularization semi-norm $|\cdot|_{B V(\Omega)}$ is replaced by the full norm.

${ }^{2}$ Working in a Hilbert space setting, Eggermont, LaRiccia, and Nashed consider weakly bounded noise perturbations and prove some interesting optimal error estimates (i.e. convergence rates for minimizers).
} 
A second property pertains to the existence of a class of clean simple signals which remain invariant under energy minimization. As noted by Chan and Esedoḡlu in [16] "It is in general desirable for image denoising algorithms to have a large class of 'noise-free' images that they leave invariant." Therefore, we ask whether the model allows for the exact reconstruction of $f_{0}$, i.e., the unique minimizer of $J$ with $f=f_{0}$ is $u=f_{0}$ for $f_{0}$ in some class of signals and with $\lambda$ sufficiently large.

In this note, we present a few simple, yet novel, remarks on these two properties. The first set of remarks shows that a certain condition (which we refer to as a BrezisLieb-type condition) on the fidelity norm is a natural condition for ensuring this notion of weak stability (see Section 3). In the second set, we remark that an elementary comparison principle yields some well-known results concerning loss of contrast for the original ROF and sufficient conditions for exact reconstruction. The latter are based upon the existence of regularization-realizing vector fields (see Section 4).

\section{Preliminaries}

In the sequel we will use $C$ to denote a constant that may vary from line to line, and from expression to expression. We let $\Omega \subset \mathbb{R}^{N}$ be an open Lipschitz domain, and use the space of functions with bounded variation on $\Omega \subset \mathbb{R}^{N}$, defined to be the subset of functions in $L^{1}(\Omega)$ whose distributional derivative is a finite Radon measure. Precisely (see, for example, [5]),

$$
B V(\Omega):=\left\{u \in L^{1}(\Omega):|D u|(\Omega)<\infty\right\}
$$

equipped with the norm $\|u\|_{B V(\Omega)}:=\|u\|_{L^{1}(\Omega)}+|D u|(\Omega)$, where

$$
|D u|(\Omega):=\sup \left\{\int_{\Omega} u(x) \operatorname{Div} \phi(x) d x: \phi \in \mathcal{C}_{c}^{1}\left(\Omega ; \mathbb{R}^{N}\right),\|\phi\|_{L^{\infty}(\Omega)} \leq 1\right\} .
$$

In what follows we use indistinguishably $|D u|(\Omega)$ and $|u|_{B V(\Omega)}$. We call $\psi \in \mathcal{C}_{c}^{1}\left(\Omega ; \mathbb{R}^{N}\right)$ a TV-realizing vector-field for $u \in B V(\Omega)$ if $\|\psi\|_{L^{\infty}(\Omega)} \leq 1$ and

$$
|u|_{B V(\Omega)}=-\int_{\Omega} u(x) \operatorname{Div} \psi(x) d x .
$$

A sequence $\left\{u_{n}\right\}$ in $B V(\Omega)$ is said to converge weakly* to $u \in B V(\Omega)$ if $u_{n} \rightarrow u$ in $L^{1}(\Omega)$ and $D u_{n} \stackrel{*}{\rightarrow} D u$ in $\Omega$. Here $D u_{n}$ and $D u$ denote the distributional derivatives which are Radon measures, and $\stackrel{*}{\rightarrow}$ stands for weak convergence of measures. It can be shown that every sequence $\left\{u_{n}\right\}$ with $\sup _{n \in \mathbb{N}}\left\|u_{n}\right\|_{B V(\Omega)}<\infty$ admits a subsequence $\left\{u_{n_{k}}\right\}$ that weakly* converges to a function $u \in B V(\Omega)$ (see [5, Theorem 3.23]).

As in [27], we denote by $G$ the dual space of the closure of $B V$ in the Schwartz class (see [27, Section 1.14] for a detailed discussion of this space and the associated $G$-norm). Here, the Schwartz class $S\left(\mathbb{R}^{n}\right)$ is

$$
S\left(\mathbb{R}^{n}\right):=\left\{f \in C^{\infty}\left(\mathbb{R}^{n}\right): \sup _{\alpha, \beta \in \mathbb{N}_{0}^{n}} \sup _{x \in \mathbb{R}^{n}}\left|x^{\alpha} D^{\beta} f(x)\right|<\infty\right\} .
$$

It turns out that $L^{2}(\Omega) \subset G$, and if $f \in B V(\Omega)$ and $g \in L^{2}(\Omega)$ then (see [27, Lemma 3])

$$
\left|\int_{\Omega} f(x) g(x) d x\right| \leq\|f\|_{B V(\Omega)}\|g\|_{G}
$$


We will use the following result of Brezis and Lieb on weakly converging sequences (see Theorem 1 in [10]).

LEMma 2.1. Let $\left\{f_{n}\right\}$ be a sequence of measurable functions which are uniformly bounded in $L^{p}(\Omega)$ for some $0<p<\infty$. If $f_{n} \rightarrow f$ pointwise almost everywhere, then

$$
\lim _{n \rightarrow \infty}\left\{\left\|f_{n}\right\|_{L^{p}(\Omega)}^{p}-\left\|f_{n}-f\right\|_{L^{p}(\Omega)}^{p}\right\}=\|f\|_{L^{p}(\Omega)}^{p} .
$$

We recall the definition and fundamental property of $\Gamma$-convergence (see [9]).

Definition 2.2. A sequence of functionals $F_{n}: X \rightarrow[-\infty,+\infty]$ defined on a metric space $X \Gamma$-converges to $F: X \rightarrow[-\infty, \infty]$, and we write $F_{n} \stackrel{\Gamma}{\rightarrow} F$, if

(i) (Lower bound) for every converging sequence $\left\{x_{n}\right\}, x_{n} \rightarrow x$ in $X$, one has $F(x) \leq \liminf _{n \rightarrow \infty} F_{n}\left(x_{n}\right)$,

and

(ii) (Upper bound) for every $x \in X$, there exists a sequence $\left\{x_{n}\right\}$ such that $x_{n} \rightarrow x$ and $\limsup _{n \rightarrow \infty} F_{n}\left(x_{n}\right)=F(x)$.

A consequence of $\Gamma$-convergence is (see [9]): If $F_{n} \stackrel{\Gamma}{\rightarrow} F$ and if $x_{n}$ is a minimizer of $F_{n}$ with $x_{n} \rightarrow x$, then

$$
x \text { is a minimizer of } F .
$$

\section{Remarks on the stability with respect to weak perturbations}

We address the stability of functionals of type (1.2) with respect to small weak ${ }^{3}$ perturbations, i.e., we consider a sequence of signals $\left\{f_{n}\right\}$ such that $f_{n}=f_{0}+h_{n}$. We assume that $h_{n} \rightarrow 0$ weakly in $\mathcal{Y}$. As a consequence of the Principle of Uniform Boundedness, $\sup _{n}\left\|h_{n}\right\|_{\mathcal{Y}} \leq C$, and we may restrict ourselves to (sub)sequences for which $\lim _{n \rightarrow \infty}\left\|h_{n}\right\|_{\mathcal{Y}}$ exists. Our analysis with a weak norm is similar in spirit to results presented in [19] for a second-order problem (see [19, Theorem 4.5]).

3.1. The Brezis-Lieb condition and convergence of minimizers. In this subsection, we consider sequences that converge with respect to some weak topologies in $\mathcal{X}$ (respectively $\mathcal{Y}$ ) such that the properties (H1)-(H3) below hold. We use the notation "쓰" for the appropriate weak convergence in the Banach space $\mathcal{X}$, and $\rightarrow$ for weak convergence in $\mathcal{Y}$. For example, if $\mathcal{X}$ is a reflexive Banach space, then "그" denotes the standard weak convergence in $\mathcal{X}$, and if $\mathcal{X}=B V(\Omega)$, then we denote by

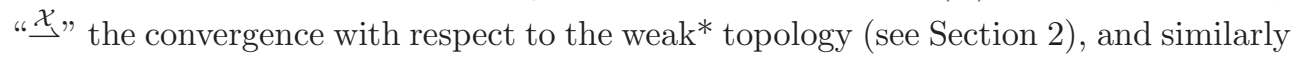
for $\mathcal{Y}$.

Here we make the following assumptions:

(H1) For every sequence $\left\{u_{n}\right\} \subset \mathcal{X}$ with $\sup _{n}\left\|u_{n}\right\|_{\mathcal{X}} \leq C$ there exists a subsequence $\left\{u_{n_{k}}\right\}$ and $u \in \mathcal{X}$ such that $u_{n_{k}} \stackrel{\mathcal{X}}{\longrightarrow} u$ in $\mathcal{X}$. The semi-norm $|\cdot|_{\mathcal{X}}$ is sequentially weakly lower semicontinuous.

\footnotetext{
${ }^{3}$ The fact that functionals (1.2) are stable with respect to small perturbations in the strong norm has been noted in [1]. If a weak fidelity measure, such as Meyer's G-norm or negative Besov-norms (see $[25,27,28]$ ), is used, then the strong condition $\left\|h_{n}\right\|_{\mathcal{Y}} \rightarrow 0$ still allows for oscillatory sequences which do not converge (even up to a subsequence) in any classical sense (see [27, Theorem 5]). See [23] for an interesting error estimate with respect to the $L^{2}$ norm in the context of bar code reconstruction.
} 
(H2) Whenever $u_{n} \stackrel{\mathcal{X}}{\rightarrow} u$ in $\mathcal{X}$, then $u_{n} \rightarrow u$ strongly in $\mathcal{Y}$.

(H3) Brezis-Lieb-type condition on $\left\{h_{n}\right\}$ : The sequence of noise samples $\left\{h_{n}\right\}$ is such that $h_{n} \rightarrow 0$ in $\mathcal{Y}$, and for every $f \in \mathcal{Y}$

$$
\|f\|_{\mathcal{Y}}^{\beta}=\lim _{n \rightarrow \infty}\left(\left\|f-h_{n}\right\|_{\mathcal{Y}}^{\beta}-\left\|h_{n}\right\|_{\mathcal{Y}}^{\beta}\right) .
$$

The hypothesis (H2) requires a compact embedding of the regularization space into the fidelity space. We first give two standard (see also [10]) examples in which the Brezis-Lieb-type condition is satisfied.

EXAMPLE 3.1.

(i) Let $\left(\mathcal{Y},(\cdot, \cdot)_{\mathcal{Y}}\right)$ be a Hilbert space, and let $\beta=2$. Then the Brezis-Lieb-type condition (H3) is satisfied for all sequences $h_{n} \rightarrow 0$ in $\mathcal{Y}$, since

$$
\begin{aligned}
& \lim _{n \rightarrow \infty}\left(\left\|f-h_{n}\right\|_{\mathcal{Y}}^{2}-\left\|h_{n}\right\|_{\mathcal{Y}}^{2}\right) \\
= & \lim _{n \rightarrow \infty}\left(\|f\|_{\mathcal{Y}}^{2}+\left\|h_{n}\right\|_{\mathcal{Y}}^{2}-2\left(f, h_{n}\right)_{\mathcal{Y}}-\left\|h_{n}\right\|_{\mathcal{Y}}^{2}\right)=\|f\|_{\mathcal{Y}}^{2} .
\end{aligned}
$$

(ii) Clearly, the Brezis-Lieb-type condition is satisfied if $\left\{h_{n}\right\}$ converges strongly. It allows, however, for some more general sequences, in particular for concentration phenomena. For $1 \leq p<\infty$ let $\left\{h_{n}\right\} \subset L^{p}(\Omega)$ converge weakly and pointwise almost everywhere to 0 . Then for all $f \in L^{p}(\Omega)$, the sequence $\left\{f-h_{n}\right\}$ converges weakly in $L^{p}(\Omega)$ and pointwise almost everywhere to $f$. Applying the Brezis-Lieb Lemma (see Lemma 2.1) to $f_{n}:=f-h_{n}$, we observe that

$$
\begin{aligned}
\|f\|_{L^{p}(\Omega)}^{p} & =\lim _{n \rightarrow \infty}\left\{\left\|f_{n}\right\|_{L^{p}(\Omega)}^{p}-\left\|f-f_{n}\right\|_{L^{p}(\Omega)}^{p}\right\} \\
& =\lim _{n \rightarrow \infty}\left\{\left\|f-h_{n}\right\|_{L^{p}(\Omega)}^{p}-\left\|h_{n}\right\|_{L^{p}(\Omega)}^{p}\right\},
\end{aligned}
$$

and hence (H3) is satisfied. A typical example of such a sequence $\left\{h_{n}\right\}$, for $p=1$, is

$$
h_{n}(x):= \begin{cases}n-n^{2} x, & 0 \leq x \leq \frac{1}{n} \\ 0, & \frac{1}{n} \leq x \leq 1\end{cases}
$$

In this subsection, define for $n \in \mathbb{N}$

$$
J_{n}(u):=|u|_{\mathcal{X}}^{\alpha}+\lambda\left\|u-f-h_{n}\right\|_{\mathcal{Y}}^{\beta} \quad \text { and } \quad J(u):=|u|_{\mathcal{X}}^{\alpha}+\lambda\|u-f\|_{\mathcal{Y}}^{\beta} .
$$

We note that hypotheses (H1)-(H3) immediately lead to the following $\Gamma$-convergence result. Recall that we restrict ourselves to sequences of noise samples $\left\{h_{n}\right\}$ for which $\lim _{n \rightarrow \infty}\left\|h_{n}\right\|_{\mathcal{Y}}^{\beta}$ exists.

THEOREM 3.2.

(i) Compactness: If (H1) holds, then for every sequence $\left\{u_{n}\right\} \subset \mathcal{X}$ with $\sup _{n \in \mathbb{N}} J_{n}\left(u_{n}\right)<\infty$, there exists a subsequence (not relabeled) such that $u_{n} \stackrel{\mathcal{X}}{\longrightarrow}$ $u$ in $\mathcal{X}$.

(ii) $\Gamma$-limit: If (H1), (H2), and (H3) hold, then setting

$$
\tilde{J}(u):=|u|_{\mathcal{X}}^{\alpha}+\lambda\|u-f\|_{\mathcal{Y}}^{\beta}+\lambda \lim _{n \rightarrow \infty}\left\|h_{n}\right\|_{\mathcal{Y}}^{\beta},
$$

we have $J_{n} \stackrel{\Gamma}{\longrightarrow} \tilde{J}$ with respect to the weak topology in $\mathcal{X}$. 
Proof. (i) By (1.3) we deduce that

$$
\begin{aligned}
\left\|u_{n}\right\|_{\mathcal{X}} & \leq C\left(\left|u_{n}\right|_{\mathcal{X}}+\left\|u_{n}\right\|_{\mathcal{Y}}\right) \leq C\left(\left|u_{n}\right|_{\mathcal{X}}+\left\|u_{n}-f-h_{n}\right\|_{\mathcal{Y}}+\left\|f+h_{n}\right\|_{\mathcal{Y}}\right) \\
& \leq C\left(\left(J_{n}\left(u_{n}\right)\right)^{1 / \alpha}+\left(J_{n}\left(u_{n}\right)\right)^{1 / \beta}+\left\|f+h_{n}\right\|_{\mathcal{Y}}\right) \leq C,
\end{aligned}
$$

where the constants $C$ depend on $\lambda, f$, and $\left\{h_{n}\right\}$. Thus, $\sup _{n \in \mathbb{N}}\left\|u_{n}\right\|_{\mathcal{X}}<\infty$ and compactness follows from (H1).

(ii) To establish the $\Gamma$-liminf of $\left\{J_{n}\right\}$, we note that by (H1) and the fact that $\alpha \geq 1$,

$$
\begin{aligned}
\liminf _{n \rightarrow \infty} J_{n}\left(u_{n}\right) & =\liminf _{n \rightarrow \infty}\left(\left|u_{n}\right|_{\mathcal{X}}^{\alpha}+\lambda\left\|u_{n}-f-h_{n}\right\|_{\mathcal{Y}}^{\beta}\right) \\
& \geq|u|_{\mathcal{X}}^{\alpha}+\lambda \liminf _{n \rightarrow \infty}\left\|u_{n}-f-h_{n}\right\|_{\mathcal{Y}}^{\beta} .
\end{aligned}
$$

Moreover, because

$$
\left(\left\|u_{n}-f-h_{n}\right\|_{\mathcal{Y}}+\left\|u_{n}-u\right\|_{\mathcal{Y}}\right)^{\beta} \geq\left\|u-f-h_{n}\right\|_{\mathcal{Y}}^{\beta},
$$

(H2), (H3), and the fact that $\beta \geq 1$ imply that

$$
\begin{aligned}
\liminf _{n \rightarrow \infty}\left\|u_{n}-f-h_{n}\right\|_{\mathcal{Y}}^{\beta} & \geq \liminf _{n \rightarrow \infty}\left\|u-f-h_{n}\right\|_{\mathcal{Y}}^{\beta} \\
& =\|u-f\|_{\mathcal{Y}}^{\beta}+\lim _{n \rightarrow \infty}\left\|h_{n}\right\|_{\mathcal{Y}}^{\beta} .
\end{aligned}
$$

Concerning the limsup inequality, if $u \in \mathcal{X}$, then setting $u_{n}:=u$ for all $n \in \mathbb{N}$, it follows again from (H3) that

$$
\lim _{n \rightarrow \infty} J_{n}(u)=|u|_{\mathcal{X}}^{\alpha}+\lambda \lim _{n \rightarrow \infty}\left\|u-f-h_{n}\right\|_{\mathcal{Y}}^{\beta}=|u|_{\mathcal{X}}^{\alpha}+\lambda\left(\|u-f\|_{\mathcal{Y}}^{\beta}+\lim _{n \rightarrow \infty}\left\|h_{n}\right\|_{\mathcal{Y}}^{\beta}\right),
$$

and thus the constant sequence $\{u\}$ is a recovery sequence.

The fundamental property (2.4) of $\Gamma$-Convergence, Theorem 3.2 yields the following corollary.

Corollary 3.3. If (H1), (H2), and (H3) hold, and if $u_{n}$ is a minimizer of $J_{n}$, then (up to a sub-sequence) $u_{n} \stackrel{\mathcal{X}}{-} u$ in $\mathcal{X}$ and $u$ is a minimizer of $J$. Additionally, the same holds true if, in place of (H2), we assume that the sequence of minimizers $\left\{u_{n}\right\}$ has a subsequence that converges strongly in $\mathcal{Y}$.

If we consider $L^{p}$-penalization in the non-Hilbert space case $p \neq 2$, the BrezisLieb-type condition does not hold true for general weakly converging sequences. In this case, we cannot expect convergence of minimizers in general. We give an example for the widely used $L^{1}$-penalization.

EXAMPLE 3.4. Let $f=0$, and let a sequence of noise samples $\left\{h_{n}\right\} \subset L^{1}(0,1)$ be given by

$$
h_{n}(x):=h(n x) \quad \text { with } \quad h(x):= \begin{cases}2, & 0<x \leq \frac{1}{3}, \\ -1, & \frac{1}{3}<x<1,\end{cases}
$$

where $h$ is extended periodically to $\mathbb{R}$ with period 1 . Consider the associated functionals

$$
J(u):=|u|_{B V(0,1)}+\lambda\|u\|_{L^{1}(0,1)} \quad \text { and } \quad J_{n}(u):=|u|_{B V(0,1)}+\lambda\left\|u-h_{n}\right\|_{L^{1}(0,1)} .
$$


The Brezis-Lieb condition (H3) is not satisfied because, e.g., for the constant function $1 \in L^{1}(0,1)$,

$$
\|1\|_{L^{1}(0,1)}=1 \neq \frac{1}{3}=\lim \left\{\left\|1-h_{n}\right\|_{L^{1}(0,1)}-\left\|h_{n}\right\|_{L^{1}(0,1)}\right\} .
$$

Clearly $u=0$ is the unique minimizer of $J$. However, for any $n \in \mathbb{N}$,

$$
J_{n}(0)=\lambda\left\|h_{n}\right\|_{L^{1}(0,1)}=\lambda\left(\frac{1}{3} \cdot 2+\frac{2}{3} \cdot 1\right)=\frac{4}{3} \lambda
$$

and

$$
J_{n}(-1)=\lambda\left\|1+h_{n}\right\|_{L^{1}(0,1)}=\lambda\left(\frac{1}{3} \cdot 3+\frac{2}{3} \cdot 0\right)=\lambda<\frac{4}{3} \lambda=J_{n}(0),
$$

so that $u=0$ is not a minimizer for any $n$. Also, if $u_{n}$ is the minimizer of $J_{n}$, then there is no subsequence $\left\{u_{n_{k}}\right\}$ with $u_{n_{k}} \rightarrow 0$ strongly in $L^{1}(0,1)$, or else

$$
\begin{aligned}
J_{n_{k}}\left(u_{n_{k}}\right) & =\left|u_{n_{k}}\right|_{B V(0,1)}+\lambda\left\|u_{n_{k}}-h_{n_{k}}\right\|_{L^{1}(0,1)} \\
& \geq \lambda\left(\left\|h_{n_{k}}\right\|_{L^{1}(0,1)}-\left\|u_{n_{k}}\right\|_{L^{1}(0,1)}\right) \\
& \geq \lambda\left(\frac{4}{3}-\left\|u_{n_{k}}\right\|_{L^{1}(0,1)}\right) \\
& >\lambda \\
& =J_{n_{k}}(-1)
\end{aligned}
$$

for large enough $n_{k} \in \mathbb{N}$, contradicting the minimality of $u_{n_{k}}$.

3.2. Convergence of minimizers in the ROF model. Here we consider the classical ROF model, i.e., for $\Omega$ an open Lipschitz domain in $\mathbb{R}^{2}$,

$$
J(u):=|u|_{B V(\Omega)}+\lambda\|u-f\|_{L^{2}(\Omega)}^{2} \quad \text { and } \quad J_{n}(u)=|u|_{B V(\Omega)}+\lambda\left\|u-f-h_{n}\right\|_{L^{2}(\Omega)}^{2} .
$$

Because $J$ is strictly convex, there exists a unique minimizer of $J$ in $B V(\Omega)$. The results in Subsection 3.1 do not directly apply to the classical ROF since (H2) is not satisfied. Under natural additional assumptions ${ }^{4}$, however, we obtain convergence of minimizers also for ROF. We outline two natural conditions which ensure that sequences $\left\{u_{n}^{*}\right\}$ of minimizers $u_{n}^{*}$ of $J_{n}$ admit subsequences that converge strongly in $L^{2}(\Omega)$ to a minimizer of $J$ : In Proposition 3.6 we note that by restricting our attention to bounded perturbations, the analogous result to Theorem 3.2 carries over. Proposition 3.7 is based upon a more general condition involving the $G$-norm, and its proof follows directly from arguments of Meyer (see [27, Section 1.14]).

For the first condition we will need the following auxiliary truncation lemma (see also [27, page 36$])$.

Lemma 3.5. Let $\Omega \subset \mathbb{R}^{2}$ and let $f \in L^{\infty}(\Omega)$. Then the minimizer $u^{*}$ of $J$ satisfies $u^{*} \in L^{\infty}(\Omega)$ and $\left\|u^{*}\right\|_{L^{\infty}(\Omega)} \leq\|f\|_{L^{\infty}(\Omega)}$.

\footnotetext{
${ }^{4}$ In general, we do not expect to find a strongly convergent subsequence. Consider $f \equiv 0, \Omega=\mathbb{R}^{2}$, and $h_{n}=n \chi_{B_{\frac{1}{n}}}(\mathbf{0})$, where $\chi_{B_{\frac{1}{n}}}(\mathbf{0})$ denotes the characteristic function of the ball with radius $\frac{1}{n}$ and center $\mathbf{0} \in \mathbb{R}^{2}$. One can readily check that for large $\lambda$ the minimizer $u_{n}^{*}$ of $J_{n}$ is $c h_{n}$, where $c=\mathcal{O}(1)$ as $n \rightarrow \infty$ (see Theorem 1 in [32], with $d=2, n=1$, and $\alpha=$ const.). Clearly, the sequence $\left\{u_{n}^{*}\right\}$ converges weakly in $L^{2}\left(\mathbb{R}^{2}\right)$ to $u \equiv 0$ as $n$ tends to infinity, but there is no strongly converging subsequence.
} 
Proof. Given $u \in B V(\Omega)$ with $\|u\|_{L^{\infty}(\Omega)}>\|f\|_{L^{\infty}(\Omega)}$ (possibly $\left.\|u\|_{L^{\infty}(\Omega)}=\infty\right)$, define $\tilde{u} \in L^{\infty}(\Omega)$ pointwise almost everywhere by

$$
\tilde{u}(x):= \begin{cases}-\|f\|_{L^{\infty}(\Omega)}, & \text { if } u(x) \leq-\|f\|_{L^{\infty}(\Omega)}, \\ u(x), & \text { if }-\|f\|_{L^{\infty}(\Omega)} \leq u(x) \leq\|f\|_{L^{\infty}(\Omega)}, \\ \|f\|_{L^{\infty}(\Omega)}, & \text { if }\|f\|_{L^{\infty}(\Omega)} \leq u(x) .\end{cases}
$$

Clearly $\|\tilde{u}\|_{L^{\infty}(\Omega)} \leq\|f\|_{L^{\infty}(\Omega)}$ and $\|\tilde{u}-f\|_{L^{2}(\Omega)}<\|u-f\|_{L^{2}(\Omega)}$. We claim that further $|\tilde{u}|_{B V(\Omega)} \leq|u|_{B V(\Omega)}$, and hence $J(\tilde{u})<J(u)$. Indeed, let $\phi_{\epsilon}$ be mollifiers, and for $a>0$ consider

$$
\tau_{a}(t):= \begin{cases}-a, & \text { if } t \leq-a \\ t, & \text { if }-a \leq t \leq a \\ a, & \text { if } t \geq a\end{cases}
$$

Let $U \subset \Omega$ be such that $|D u|(\partial U)=0$. Then we have

$$
|D u|(\Omega) \geq|D u|(U)=\lim _{\epsilon \rightarrow 0} \int_{U}\left|\nabla\left(\phi_{\epsilon} * u\right)\right| \geq \limsup _{\epsilon \rightarrow 0} \int_{U}\left|\nabla\left(\tau_{a} \circ\left(\phi_{\epsilon} * u\right)\right)\right| \geq\left|D\left(\tau_{a} \circ u\right)\right|(U),
$$

where the equality follows from Proposition 3.7 in [5], and in the last inequality we used the fact that $\tau_{a} \circ\left(\phi_{\epsilon} * u\right) \rightarrow \tau_{a} \circ u$ in $L^{1}(U)$ together with lower semicontinuity of the total variation. Letting $U \nearrow \Omega$, we obtain $|D u|(\Omega) \geq\left|D\left(\tau_{a} \circ u\right)\right|(\Omega)$. It follows that, if $u^{*}$ is a minimizer of $J$, then $u^{*} \in L^{\infty}(\Omega)$ and $\left\|u^{*}\right\|_{L^{\infty}(\Omega)} \leq\|f\|_{L^{\infty}(\Omega)}$.

Proposition 3.6. If $f \in L^{\infty}(\Omega)$ and if the sequence of noise samples $\left\{h_{n}\right\}$ is uniformly bounded, i.e.,

$$
\sup _{n \in \mathbb{N}}\left\|h_{n}\right\|_{L^{\infty}(\Omega)}<+\infty
$$

and converges weakly to 0 in $L^{2}(\Omega)$, then minimizers of $J_{n}$ converge to minimizers of $J$ weakly* in $B V(\Omega)$.

Proof. In view of Corollary 3.3 and Example 3.1, it remains to show that for a sequence of minimizers $\left\{u_{n}\right\}$ of $J_{n}$, there exists a subsequence that converges strongly to $u$ in $L^{2}(\Omega)$. By the compact embedding $B V(\Omega) \subset L^{1}(\Omega)$, there exists a subsequence (not relabeled) such that $u_{n} \rightarrow u$ strongly in $L^{1}(\Omega)$ for some $u \in B V(\Omega)$. By Lemma 3.5, $\sup _{n}\left\|u_{n}\right\|_{L^{\infty}(\Omega)} \leq \sup _{n}\left\|f+h_{n}\right\|_{L^{\infty}(\Omega)}<+\infty$, and thus $u \in L^{\infty}(\Omega)$ and

$$
\left\|u_{n}-u\right\|_{L^{2}(\Omega)}^{2} \leq\left\|u_{n}-u\right\|_{L^{\infty}(\Omega)}\left\|u-u_{n}\right\|_{L^{1}(\Omega)} \rightarrow 0 .
$$

Hence $u_{n} \rightarrow u$ strongly in $L^{2}(\Omega)$.

Proposition 3.7. Let $f \in L^{2}(\Omega)$, and let $\left\{h_{n}\right\}$ be a sequence of noise samples such that $h_{n} \rightarrow 0$ in $L^{2}(\Omega)$ and $\left\|h_{n}\right\|_{G} \rightarrow 0$. Let $u_{n} \in B V(\Omega)$ be a minimizer of $J_{n}$. Then there exists a subsequence (not relabeled) $\left\{u_{n}\right\}$ and $u \in B V(\Omega)$ such that $u_{n}{ }^{*} u$ in $B V(\Omega)$, and $u$ is the minimizer of $J$.

Proof. By Theorem 3.2 (i), there exists a subsequence (not relabeled) such that $u_{n} \stackrel{*}{\rightarrow} u$ for some $u \in B V(\Omega)$. For all $v \in B V(\Omega)$, we have $J_{n}\left(u_{n}\right) \leq J_{n}(v)$, i.e., 


$$
\begin{aligned}
& \left|u_{n}\right|_{B V(\Omega)}+\lambda\left\|u_{n}-f\right\|_{L^{2}(\Omega)}^{2}-2 \lambda\left(u_{n}, h_{n}\right)_{L^{2}(\Omega)} \\
\leq & |v|_{B V(\Omega)}+\lambda\|v-f\|_{L^{2}(\Omega)}^{2}-2 \lambda\left(v, h_{n}\right)_{L^{2}(\Omega)} .
\end{aligned}
$$

By (2.3), we have $\left(u_{n}, h_{n}\right)_{L^{2}(\Omega)} \leq\left\|u_{n}\right\|_{B V(\Omega)}\left\|h_{n}\right\|_{G}$, hence

$$
\begin{aligned}
& \left|u_{n}\right|_{B V(\Omega)}\left(1-2 \lambda\left\|h_{n}\right\|_{G}\right)-2 \lambda\left\|u_{n}\right\|_{L^{1}(\Omega)}\left\|h_{n}\right\|_{G}+\lambda\left\|u_{n}-f\right\|_{L^{2}(\Omega)}^{2} \\
\leq & |v|_{B V(\Omega)}+\lambda\|v-f\|_{L^{2}(\Omega)}^{2}-2 \lambda\left(v, h_{n}\right)_{L^{2}(\Omega)} .
\end{aligned}
$$

Since $h_{n} \rightarrow 0$ in $L^{2}(\Omega),\left\|h_{n}\right\|_{G} \rightarrow 0$, and $\left\|u_{n}\right\|_{L^{1}(\Omega)} \rightarrow\|u\|_{L^{1}(\Omega)}$, we may take liminf ${ }_{n \rightarrow \infty}$ above and use Fatou's Lemma (after a possible extraction of a subsequence) to conclude that

$$
|u|_{B V(\Omega)}+\lambda\|u-f\|_{L^{2}(\Omega)}^{2} \leq|v|_{B V(\Omega)}+\lambda\|v-f\|_{L^{2}(\Omega)}^{2} .
$$

Thus $u$ is a minimizer of $J$.

So far, we proved convergence of minimizers of the disturbed functionals $J_{n}$ to minimizers of $J$, although in general a minimizer of $J$ is not a minimizer of $J_{n}$. However, in the ROF model the trivial minimizer has the following higher stability property, which follows directly from [27, Lemma 4].

Proposition 3.8. If $\|f\|_{G}<\frac{1}{2 \lambda}$, then $u \equiv 0$ is the minimizer of $J$, and for any sequence $\left\{h_{n}\right\}$ with $h_{n} \rightarrow 0$ weakly in $L^{2}(\Omega)$ and $\left\|h_{n}\right\|_{G} \rightarrow 0$, there exists a $n_{0} \in \mathbb{N}$ such that, for all $n \geq n_{0}$, the function $u \equiv 0$ is a minimizer of $J_{n}$, where

$$
J(u):=|u|_{B V(\Omega)}+\lambda\|u-f\|_{L^{2}(\Omega)}^{2}, \quad J_{n}(u):=|u|_{B V(\Omega)}+\lambda\left\|u-f-h_{n}\right\|_{L^{2}(\Omega)}^{2} .
$$

We note that the stability properties presented in Theorem 3.2, Corollary 3.3, and propositions 3.6 and 3.7 do not depend on the scaling exponent $\alpha>1$ of the regularization term. The higher stability of the trivial minimizer, however, depends heavily on $\alpha$, and fails in general if $\alpha>1$. Indeed, set

$$
h_{n}(x):=h(n x), \quad \text { where } \quad h(x):= \begin{cases}-1, & 0 \leq x \leq \frac{1}{2}, \\ +1, & \frac{1}{2} \leq x \leq 1,\end{cases}
$$

and $h$ is extended periodically to $\mathbb{R}$ with period 1 , and consider

$$
J_{n}(u):=|u|_{B V(0,1)}^{2}+\lambda\left\|u-h_{n}\right\|_{L^{2}(0,1)}^{2} .
$$

Then $J_{n}(0)=\lambda\left\|h_{n}\right\|_{L^{2}(0,1)}^{2}=\lambda$. Now consider the competitor functions $u_{n}(x):=c_{n} h_{n}$ with $c_{n}:=\frac{\lambda}{n^{2}+\lambda}$. We have

$$
J_{n}\left(u_{n}\right)=\frac{\lambda^{2} n^{2}}{\left(n^{2}+\lambda\right)^{2}}+\lambda \frac{n^{4}}{\left(n^{2}+\lambda\right)^{2}}=\frac{\lambda n^{2}+n^{4}}{\left(n^{2}+\lambda\right)^{2}} \lambda<\lambda=J(0),
$$

so that $u \equiv 0$ is not a minimizer of $J_{n}$ for any $n \in \mathbb{N}$.

REMARK 3.9. In this note we have modelled the noise by deterministic oscillatory perturbations. Future developments, still within the scope of deterministic perturbation, may consider other fidelity norms suitable for different noise samples. While $L^{2}$ 
and $L^{1}$ are well motivated by additive Gaussian or Laplace noise, current research in this area uses different data fidelities motivated as log-likelihoods of statistical noise models. For example, Poisson noise leads to a Kullback-Leibler divergence [26], while signal-dependent noise leads to a weighted $L^{2}$-norm [29]. An important direction that this work may take is to move away from the deterministic setting and consider stochastic noise perturbations.

4. Remarks on the invariance for clean, simple signals: exact reconstruction

We consider functionals $J$ of the general form (1.2). We say that $f$ is exactly reconstructable for some $\lambda>0$, if the unique minimizer of $J$ is $f$. Fix $f \in \mathcal{X}$. We first note the following elementary comparison principle: $f$ is a minimizer of $J$, i.e. $J(f) \leq J(u)$ for all $u \neq f$, if and only if

$$
|f|_{\mathcal{X}}^{\alpha} \leq|u|_{\mathcal{X}}^{\alpha}+\lambda\|u-f\|_{\mathcal{Y}}^{\beta} \quad \text { for all } \quad u \neq f
$$

or, equivalently,

$$
\lambda \geq \sup _{u \neq f} \frac{|f|_{\mathcal{X}}^{\alpha}-|u|_{\mathcal{X}}^{\alpha}}{\|u-f\|_{\mathcal{Y}}^{\beta}}
$$

The inequality in (4.1) is strict if and only if $f$ is the unique minimizer of $J$.

Proposition 4.1 (Scaling and the Failure of Exact Reconstructibility). If $\beta>1$ and $|f|_{\mathcal{X}} \neq 0$, then $f$ is not exactly reconstructable for any finite $\lambda>0$.

Proof. For $0<\epsilon<1$ define the test functions $u_{\epsilon}:=(1-\epsilon) f$. Then

$$
\sup _{u \neq f} \frac{|f|_{\mathcal{X}}^{\alpha}-|u|_{\mathcal{X}}^{\alpha}}{\|f-u\|_{\mathcal{Y}}^{\beta}} \geq \sup _{0<\epsilon<1} \frac{\left(1-(1-\epsilon)^{\alpha}\right)|f|_{\mathcal{X}}^{\alpha}}{\epsilon^{\beta}\|f\|_{\mathcal{Y}}^{\beta}}=\infty
$$

since $1-(1-\epsilon)^{\alpha}=\mathcal{O}(\epsilon)$ as $\epsilon \rightarrow 0$.

Note that, as a consequence of this result, regardless of the choice of regularization, only the trivial images with $|f|_{\mathcal{X}}=0$ are invariant if one uses as fidelity norm the $L^{2}$-norm squared. We recall that in contrast, the $L^{2}$-norm squared satisfies the Brezis-Lieb condition, and hence yields stability of minimizers with respect to weak perturbation. It is well-known that minimizers of the ROF functional (1.4) possess a loss of contrast, and indeed the only clean image which is left invariant is $f \equiv 0$ (see $[16,32])$.

The next corollary addresses the borderline case $\beta=1$. In particular, for $L^{p}$ fidelity terms it has been noted that a signal $f$ is left invariant by energy minimization (for $\lambda$ sufficiently large) if and only if there exists a suitably regular, TV-realizing vector field (see $[27,16,30,7,18]$ ). Let us first note here that the sufficiency of such a vector field immediately follows from our comparison estimate (4.1). Let $1 \leq p<\infty$, let $\mathcal{Y}:=L^{p}(\Omega)$, and consider

$$
J^{(\lambda)}(u):=|u|_{\mathcal{X}}+\lambda\|u-f\|_{L^{p}(\Omega)} .
$$

Here we use the notation $J^{(\lambda)}$ to highlight the dependence on the regularization parameter $\lambda$. We set $p *:=\frac{p}{p-1}$ if $p \neq 1$, and $p *=\infty$ if $p=1$. 
COROLlaRY 4.2 (Exact Reconstruction and Regularization-realizing Vector Fields). Suppose there is a function space $\mathcal{F}$ such that

$$
|u|_{\mathcal{X}}=\sup _{\phi \in \mathcal{F}} \int_{\Omega} u(x) \phi(x) d x \quad \text { for all } u \in \mathcal{X} .
$$

If there exists a $\phi_{f} \in \mathcal{F} \cap L^{p *}(\Omega)$ such that

$$
|f|_{\mathcal{X}}=\int_{\Omega} f(x) \phi_{f}(x) d x
$$

then for $\lambda \geq\left\|\phi_{f}\right\|_{L^{p *}(\Omega)}$ the signal $f$ is a minimizer of $J^{(\lambda)}$ among all $u \in \mathcal{X}$.

Proof. For $u \in \mathcal{X}$, using Hölder's inequality we estimate

$$
\begin{aligned}
\frac{|f|_{\mathcal{X}}-|u|_{\mathcal{X}}}{\|f-u\|_{L^{p}(\Omega)}} & \leq \frac{\int_{\Omega}\left[(f(x)-u(x)) \phi_{f}(x)\right] d x}{\|f-u\|_{L^{p}(\Omega)}} \\
& \leq \frac{\|f-u\|_{L^{p}(\Omega)}\left\|\phi_{f}\right\|_{L^{p *}(\Omega)}}{\|f-u\|_{L^{p}(\Omega)}} \\
& =\left\|\phi_{f}\right\|_{L^{p *}(\Omega)} .
\end{aligned}
$$

It now suffices to apply (4.1).

A well-studied special case of Corollary 4.2 is TV-regularization (see [27, 30, $16,7,18])$, where $\mathcal{X}:=B V(\Omega), \mathcal{F}:=\left\{\operatorname{Div}(v): v \in \mathcal{C}_{c}^{1}\left(\Omega ; \mathbb{R}^{N}\right),\|v\|_{L^{\infty}(\Omega)} \leq 1\right\}$, and $\mathcal{Y}:=L^{1}(\Omega)$. Then $f$ is reconstructable if there exists a vector field $v \in L^{1}(\Omega)$ with $\operatorname{Div}(v) \in \mathcal{F}$ such that

$$
|f|_{B V(\Omega)}=\int_{\Omega} f \operatorname{Div} v d x
$$

The existence of the associated vector field is often also a necessary condition, (see $[18,30]$ for a proof using convex analysis). In fact, this regularization-realizing condition can readily be formulated as a subgradient relation, and (4.2) is often used as a source condition in error estimation (see [12]).

Lastly, we show how the comparison estimate (4.1) can be used, in the case in which $\mathcal{X}:=B V(\Omega)$, to gain insight into when a smooth signal is reconstructable or not. Suppose that we are given a signal $f \in W^{1, \infty}(\Omega)$, i.e., $f \in L^{1}(\Omega)$ and its first weak derivative exists in $L^{\infty}(\Omega)$. Integration by parts in (4.2) implies that a TV-realizing vector field exists if and only if there is a function $g \in \mathcal{C}_{c}^{1}\left(\Omega ; \mathbb{R}^{N}\right)$ with $\|g\|_{L^{\infty}(\Omega)} \leq 1$ and $g=\frac{\nabla f}{|\nabla f|}$ on the support of $\nabla f$ (see [27]). If $\Omega \subset \mathbb{R}$ then the existence of such a function $g \in \mathcal{C}_{c}^{1}(\Omega ; \mathbb{R})$ depends crucially on the points at which the derivative $f^{\prime}$ changes sign, i.e., the extremal points of $f$. In particular, any function $f$ with a strict local extremum is not reconstructable. We give an elementary proof of this statement which also provides an explicit competitor for given $\lambda<\infty$.

Corollary 4.3. Let $\Omega \subset \mathbb{R}$ be closed, bounded, and connected, and let $f: \Omega \rightarrow \mathbb{R}$ be absolutely continuous. If $f$ has a strict local extremum, then $f$ is not a minimizer of $J^{(\lambda)}$ for any $1 \leq p<\infty$ and any $\lambda>0$.

Proof. Fix $p \in[1, \infty)$ and $\lambda>0$. Without loss of generality, we may assume that $f$ has a strict local minimum at $x_{0}=0, f(0)=0$, and $f(x)>f(0)=0$ for all $x \in(-\epsilon, \epsilon)$. 
Suppose first that $x_{0}$ is an interior point of $\Omega$. Since $f$ is continuous at 0 and $f(0)=0$, for $n \geq N$, there are $x \in(-\epsilon, 0)$ and $y \in(0, \epsilon)$ such that $f(x)=\frac{1}{n}$ and $f(y)=\frac{1}{n}$. Define $y_{n}:=\inf \left\{y \in(0, \epsilon): f(y)=\frac{1}{n}\right\}$. Then by continuity of $f$ we have $y_{n}>0$, and $y_{n} \rightarrow 0^{+}$as $n \rightarrow \infty$. Analogously, set $x_{n}:=\sup \left\{x \in(-\epsilon, 0): f(x)=\frac{1}{n}\right\}$. Then $x_{n}<0$, and $x_{n} \rightarrow 0^{-}$ as $n \rightarrow \infty$. Define the test functions

$$
u_{n}(x):= \begin{cases}f(x), & x \in \Omega \backslash\left(x_{n}, y_{n}\right), \\ \frac{1}{n}, & x \in\left(x_{n}, y_{n}\right)\end{cases}
$$

We note that by construction,

$$
\left\|f-u_{n}\right\|_{L^{p}(\mathbb{R})}=\left(\int_{x_{n}}^{y_{n}}\left|\frac{1}{n}-f(x)\right|^{p} d x\right)^{1 / p} \leq \frac{1}{n}\left(y_{n}-x_{n}\right)^{1 / p},
$$

and

$$
\int_{x_{n}}^{y_{n}}\left|f^{\prime}\right| d x \geq\left|\int_{0}^{y_{n}} f^{\prime}(x) d x\right|=\frac{1}{n}
$$

The latter implies

$$
|f|_{B V(\Omega)} \geq\left|u_{n}\right|_{B V(\Omega)}+\frac{1}{n} .
$$

Thus, by (4.4) and (4.3) we deduce that

$$
\sup _{n \geq N} \frac{|f|_{B V(\mathbb{R})}-\left|u_{n}\right|_{B V(\mathbb{R})}}{\left\|f-u_{n}\right\|_{L^{p}(\mathbb{R})}} \geq \sup _{n \geq N} \frac{1 / n}{1 / n\left(y_{n}-x_{n}\right)^{1 / p}} \geq \sup _{n \geq N} \frac{1}{\left(y_{n}-x_{n}\right)^{1 / p}}=\infty
$$

since $x_{n} \rightarrow 0$ and $y_{n} \rightarrow 0$. The claim follows in view of (4.1).

Suppose now that $x_{0} \in \partial \Omega$. Without loss of generality, we may assume that $x_{0}:=0$, $(0, \epsilon) \subset \Omega, \mathbb{R}_{-} \cap \Omega=\emptyset$, and $f(x)>f(0)$ for all $x \in(0, \epsilon)$. Then there is a sequence $\left\{y_{n}\right\}$ such that, for $n \geq N$, we have $0<y_{n}<\epsilon, f\left(y_{n}\right)=\frac{1}{n}$, and $f(x)<\frac{1}{n}$ for all $x \in\left(0, y_{n}\right)$. We proceed as above. Define the test functions

$$
u_{n}(x):= \begin{cases}f(x), & x \in \Omega \backslash\left(0, y_{n}\right), \\ \frac{1}{n}, & x \in\left(0, y_{n}\right) .\end{cases}
$$

Then $|f|_{B V(\Omega)} \geq\left|u_{n}\right|_{B V(\Omega)}+\frac{1}{n}$ and $\left\|f-u_{n}\right\|_{L^{p}(\Omega)} \leq \frac{1}{n} y_{n}^{1 / p}$, and we conclude as in (4.5).

We conclude by noting that in a series of papers (see $[2,3,4]$ ), Allard gives a detailed analysis of properties of minimizers for total variation based functionals. In particular, he proves a regularity result for the level sets and gives very restrictive properties of the minimizers in the case in which the input signal $f$ is a characteristic function $\chi_{S}$ of a compact, convex set $S \subset \mathbb{R}^{2}$ with non-empty interior and $\left|\chi_{S}\right|_{B V}<\infty$. In particular, Theorem 1.1 in [3] yields as a corollary that for $L^{2}$-squared fidelity no such indicator function can be a "local" minimizer of

$$
J^{(\lambda)}(u):=|u|_{B V\left(\mathbb{R}^{2}\right)}+\lambda\left\|u-\chi_{S}\right\|_{L^{2}\left(\mathbb{R}^{2}\right)}^{2} .
$$

Indeed, adopting the notation of [3], set $\mathcal{F}:=\left\{u \in L^{1}\left(\mathbb{R}^{2}\right) \cap L^{\infty}\left(\mathbb{R}^{2}\right): u \geq 0\right\}$, and let

$$
m_{\lambda}^{\text {loc }}:=\left\{u \in \mathcal{F}:|u|_{B V}<\infty \text { and } J^{(\lambda)}(u) \leq J^{(\lambda)}(g) \text { for all } g \in \mathcal{F}\right. \text { for which }
$$


there is $K \subset \mathbb{R}^{2}$ compact s.t. $g(x)=u(x)$ for a.e. $\left.x \in \mathbb{R}^{2} \backslash K\right\}$.

For $0<y<1$ let $W_{y}:=\left\{x \in \mathbb{R}^{2}: \operatorname{dist}\left(x, C_{1 /(2 \lambda(1-y))}\right) \leq \frac{1}{2 \lambda(1-y)}\right\}$, where $C_{r}:=\{x \in S$ : $\left.B_{r}(x) \subset S\right\}$, and introduce the function

$$
\Psi(y):=\left|\chi_{W_{y}}\right|_{B V}+2 \lambda(y-1)\left|W_{y}\right|,
$$

where $\left|W_{y}\right|$ denotes the 2-dimensional Lebesgue measure of $W_{y}$ in $\mathbb{R}^{2}$. Set $I:=\{y \in$ $\left.(0,1): W_{y} \neq \emptyset\right\}, I_{\mp}:=\{y \in I: \Psi(y) \lessgtr 0\}, I_{0}:=\{y \in I: \Psi(y)=0\}$, and define

$$
M:= \begin{cases}\{0\}, & \text { if } I_{-} \cup I_{0}=\emptyset, \\ \left\{\sup I_{-}\right\}, & \text {if } I_{-} \neq \emptyset \text { and } I_{0}=\emptyset, \\ \left\{y: \inf I_{0} \leq y \leq \sup I_{0}\right\}, & \text { if } I_{0} \neq \emptyset .\end{cases}
$$

Let $\theta:=\sup \left\{r>0: \exists x \in S\right.$ s.t. $\left.B_{r}(x) \subset S\right\}$. Note that if $\frac{1}{2 \lambda}>\theta$, then $\frac{1}{2 \lambda(1-y)}>\theta$ for all $y \in(0,1)$, which implies $C_{1 /(2 \lambda(1-y))}=\emptyset$, and thus $W_{y}=\emptyset$ for all $y \in(0,1)$.

We have the following theorem (see [3, Theorem 1.1]).

Theorem 4.1. $u \in m_{\lambda}^{\text {loc }}$ if and only if for some $Y \in M$ we have, for $\mathcal{L}^{1}$ a.e. $y \in(0,1)$,

$$
\{u>y\}= \begin{cases}W_{y}, & \text { if } \quad 0<y \leq Y \\ \emptyset, & \text { if } \quad Y<y\end{cases}
$$

COROLlary 4.2. Let $S$ be a compact, convex set of finite perimeter with non-empty interior. Then $\chi_{S} \notin m_{\lambda}^{\text {loc }}$.

Proof. Assume that $\chi_{S} \in m_{\lambda}^{\text {loc }}$. If $W_{y}=\emptyset$ for all $y \in(0,1)$, then $\emptyset=I=I_{-}=$ $I_{+}=I_{0}$, and $Y=0$, and then by Theorem 4.1 we have that $S=\left\{\chi_{S}>y\right\}=\emptyset$ for a.e. $y \in(0,1)$, which is a contradiction. If there exists $y \in(0,1)$ such that $W_{y} \neq \emptyset$ then by Theorem 4.1, and because $S=\left\{\chi_{S}>y\right\}$, we have $Y=1$ and $W_{y}=S$ for all $0<y<1$. Thus, by definition of $M$, either we have $1=\sup I_{-}$or $I_{0}=\{1\}$, and the latter is not possible because $I_{0} \subset I \subset(0,1)$. Hence $\sup I_{-}=1$, and so there is a sequence $\left\{y_{k}\right\} \subset(0,1)$ with $y_{k} \rightarrow 1$ and $0 \geq \Psi\left(y_{k}\right) \rightarrow\left|\chi_{S}\right|_{B V}$ as $k \rightarrow \infty$, which is a contradiction. 口

We note that Theorem 4.1 (see [3]) generalizes Proposition 3.8 (see [27]) in what concerns not only the minimizers but also the local minimizers if the input signal is the characteristic function of a compact, convex set. Consider, for example, $S:=B_{1}(0)$. It can be shown that $\left\|\chi_{B_{1}(0)}\right\|_{G}=\frac{1}{2}$ (see [27, Lemma 6]). Suppose that $\frac{1}{2 \lambda}>\left\|\chi_{B_{1}(0)}\right\|_{G}=$ $\frac{1}{2}$, i.e., $\lambda<1$. Then Proposition 3.8 asserts that $u \equiv 0$ is the only minimizer of $J^{(\lambda)}$. We show that Theorem 4.1 yields more, namely that the only local minimizer is $u \equiv 0$. Indeed,

$$
\left\{x \in \mathbb{R}^{2}: \operatorname{dist}\left(x, C_{r}\right) \leq r\right\}= \begin{cases}B_{1}(0), & \text { if } r \leq 1 \\ \emptyset, & \text { else }\end{cases}
$$

Because $\frac{1}{2 \lambda(1-y)} \leq 1$ if and only if $y \leq 1-\frac{1}{2 \lambda}$, we thus have

$$
W_{y}= \begin{cases}B_{1}(0), & \text { if } y \leq 1-\frac{1}{2 \lambda} \\ \emptyset, & \text { else }\end{cases}
$$


and in particular $I=\left(0,1-\frac{1}{2 \lambda}\right)$. Now,

$$
\Psi(y):=\left|\chi_{W_{y}}\right|_{B V}+2 \lambda(y-1)\left|W_{y}\right|=2 \pi+2 \lambda(y-1) \pi>0 \quad \text { for all } y \in I
$$

because $\lambda<1$. Hence, $I_{-}=I_{0}=\emptyset$, which implies $M=\{0\}$, and therefore $Y=0$. By Theorem 4.1 we conclude that $u \equiv 0$ is the only element in $m_{\lambda}^{\text {loc }}$. In particular, $u \equiv 0$ is the only minimizer of $J^{(\lambda)}$, as follows from Proposition 3.8.

In cases where signals $f$ are not reconstructible (such as the case for ROF), an interesting question raised in [27] is whether or not the minimizer is $c f$ for $c<1$. This has recently been treated in [8] where a connection is made to some interesting eigenvalue problems.

Acknowledgments. We would like to thank both the anonymous referees and M. Burger for useful comments on the manuscript. The research of RC was partly supported by an NSERC (Canada) Discovery Grant. The research of IF was partially funded by the National Science Foundation under Grant No. DMS-0905778. The research of BZ was funded by a postdoctoral fellowship of the National Science Foundation under Grant No. DMS-0905778. IF and BZ warmly thank the Center for Nonlinear Analysis (NSF Grant No. DMS-0635983) for its support during the preparation of this paper.

\section{REFERENCES}

[1] R. Acar and C.R. Vogel, Analysis of bounded variation penalty methods for ill-posed problems, Inv. Prob., 10, 1217-1229, 1994.

[2] W.K. Allard, Total variation regularization for image denoising. I. Geometric theory, SIAM J. Math. Anal., 39, 1150-1190, 2007/2008.

[3] W.K. Allard, Total variation regularization for image denoising. II. Examples, SIAM J. Imaging Sci., 1, 400-417, 2008.

[4] W.K. Allard, Total variation regularization for image denoising. III. Examples, SIAM J. Imaging Sci., 2, 532-568, 2009.

[5] L. Ambrosio, N. Fusco, and D. Pallara, Functions of Bounded Variation and Free Discontinuity Problems, Oxford University Press, Oxford, 2000.

[6] G. Aubert and P. Kornprobst, Mathematical Problems in Image Processing, Appl. Math. Series 147, Springer, 2002.

[7] M. Benning and M. Burger, Error estimates for general fidelities, Electronic Transactions of Numerical Analysis, 38, 44-68, 2011.

[8] M. Benning and M. Burger, Ground states and singular vectors of convex variational regularization methods, Meth. Appl. Anal., to appear.

[9] A. Braides, $\Gamma$-convergence for Beginners, Oxford Lecture Series in Mathematics and its Applications, First Edition, Oxford University Press, Oxford, 22, 2002.

[10] $\mathrm{H}$. Brezis and E. Lieb, A relation between pointwise convergence of functions and convergence of functionals, Proc. of the AMS, 88(3), 486-490, 1983.

[11] A. Buades, B. Coll, and J.M. Morel, A review of image denoising algorithms, with a new one, Multiscale Model. Simul., 4, 490-530, 2005.

[12] M. Burger and S. Osher, Convergence rates of convex variational regularization, Inv. Prob., 20, 1411-1421, 2004.

[13] V. Caselles, A. Chambolle, and M. Novaga, The discontinuity set of solutions of the TV denoising problem and some extensions, Multiscale Model. Simul., 3, 879-894, 2007.

[14] V. Caselles, A. Chambolle, and M. Novaga, Regularity for solutions of the total variation denoising problem, Rev. Mat. Iberoam., 27, 233-252, 2011.

[15] A. Chambolle, An algorithm for total variation minimization and applications, J. Math. Imag. Vis., 20, 89-97, 2004.

[16] T.F. Chan and S. Esedoglu, Aspects of total variation regularized $L^{1}$ function approximation, SIAM J. Appl. Math., 65, 1817-1837, 2005.

[17] T.F. Chan and J. Shen, Image Processing and Analysis, SIAM, PA, 2005.

[18] R. Choksi, Y. van Gennip, and A. Oberman, Anisotropic total variation regularized $L^{1}$ - 
approximation and denoising/deblurring of $2 D$ bar codes, Inv. Prob. Imag., 5(3), 591-617, 2011.

[19] G. Dal Maso, I. Fonseca, G. Leoni, and M. Morini, A higher order model for image restoration: The one-dimensional case, SIAM J. Math. Anal., 40(6), 2351-2391, 2009.

[20] D. Dobson and F. Santosa, Recovery of blocky images from noisy and blurred data, SIAM Appl. Math., 56, 1181-1198, 1996.

[21] P.P.B. Eggermont, V.N. LaRiccia, and M.Z. Nashed, On weakly bounded noise in ill-posed problems, Inv. Prob., 25, 115018, 2009.

[22] S. Esedoḡlu and S.J. Osher, Decomposition of images by the anisotropic Rudin-Osher-Fatemi model, Comm. Pure Appl. Math., 57(12), 1609-1626, 2004.

[23] S. Esedoğlu and F. Santosa, Error estimates for a bar code reconstruction method, Disc. Cont. Dyn. Sys. B, 17(6), 1889-1902, 2012.

[24] T. Goldstein and S. Osher, The split Bregman method for $L^{1}$-regularized problems, SIAM J. Imaging Sci., 2, 323-343, 2009.

[25] A. Haddad and Y. Meyer, An Improvement of Rudin-Osher-Fatemi model, Appl. Comput. Harmonic Anal., 22, 319-334, 2007.

[26] S. Kullback and R.A. Leibler, On information and sufficiency, Annal. Math. Stat., 22(1), 79-86, 1951.

[27] Y. Meyer, Oscillating Patterns in Image Processing and Nonlinear Evolution Equations, University Lecture Series, American Mathematical Society, Providence, RI, 22, 2001.

[28] S. Osher, A. Solé, and L. Vese, Image decomposition and restoration using total variation minimization and the $H^{-1}$ - Norm, Multiscale Model. Simul., 1(3), 349-370, 2003.

[29] A. Repetti, E. Chouzenoux, and J.C. Pesquet, A Penalized Weighted Least Squares Approach for Restoring Data Corrupted with Signal-Dependent Noise, 20th European Signal Processing Conference (EUSIPCO 2012), Bucarest, 2012.

[30] W. Ring, Structural properties of solutions to total variation regularization problems, M2AN Math. Model. Numer. Anal., Otmar Scherzer, 34(4), 799-810, 2000.

[31] L. Rudin, S. Osher, and E. Fatemi, Nonlinear total variation based noise removal algorithms, Physica D, 60, 259-268, 1992.

[32] D. Strong and T. Chan, Edge-preserving and scale-dependent properties of total variation regularization, Inv. Prob., 19, 165-187, 2003.

[33] A.N. Tikhonov and V.Y. Arsenin, Solutions of Ill-Posed Problems, Winston and Sons, 1977.

[34] L.Vese, A study in the BV space of a denoising-debluring variational problem, Appl. Math. Optim., 44, 131-161, 2001. 